\title{
Probiotic/prebiotic supplementation of antiretrovirals improves gastrointestinal immunity in SIV-infected macaques
}

Nichole R. Klatt, ${ }^{1}$ Lauren A. Canary, ${ }^{1}$ Xiaoyong Sun, ${ }^{2}$ Carol L. Vinton, ${ }^{1}$ Nicholas T. Funderburg, David R. Morcock, ${ }^{4}$ Mariam Quiñones, ${ }^{1}$ Clayton B. Deming, ${ }^{5}$ Molly Perkins, ${ }^{1}$ Daria J. Hazuda, ${ }^{6}$ Michael D. Miller, ${ }^{7}$ Michael M. Lederman, ${ }^{3}$ Julie A. Segre, ${ }^{5}$ Jeffrey D. Lifson, ${ }^{4}$ Elias K. Haddad, ${ }^{2}$ Jacob D. Estes, ${ }^{4}$ and Jason M. Brenchley ${ }^{1}$

\begin{abstract}
${ }^{1}$ National Institute of Allergy and Infectious Diseases (NIAID), NIH, Bethesda, Maryland, USA. ${ }^{2}$ VGTI-Florida, Port St. Lucie, Florida, USA. ${ }^{3}$ Division of Infectious Diseases, Case Western Reserve University, Cleveland, Ohio, USA. ${ }^{4}$ SAIC Frederick Inc.,

Frederick National Laboratory for Cancer Research, Frederick, Maryland, USA. ${ }^{5}$ National Human Genome Research Institute (NHGRI), NIH, Bethesda, Maryland, USA. ${ }^{6}$ Merck Research Labs, West Point, Pennsylvania, USA. ${ }^{7}$ Gilead Sciences Inc., Foster City, California, USA.
\end{abstract}

\begin{abstract}
HIV infection results in gastrointestinal (GI) tract damage, microbial translocation, and immune activation, which are not completely ameliorated with suppression of viremia by antiretroviral (ARV) therapy. Furthermore, increased morbidity and mortality of ARV-treated HIV-infected individuals is associated with these dysfunctions. Thus, to enhance GI tract physiology, we treated SIV-infected pigtail macaques with ARVs, probiotics, and prebiotics or with ARVs alone. This synbiotic treatment resulted in increased frequency and functionality of GI tract APCs, enhanced reconstitution and functionality of $\mathrm{CD}^{+} \mathrm{T}$ cells, and reduced fibrosis of lymphoid follicles in the colon. Thus, ARV synbiotic supplementation in HIV-infected individuals may improve GI tract immunity and thereby mitigate inflammatory sequelae, ultimately improving prognosis.
\end{abstract}

\section{Introduction}

Antiretroviral (ARV) treatment of HIV-infected individuals improves their prognosis; however, ARV-treated individuals still have increased morbidity and mortality compared with uninfected individuals (1). During progressive HIV infection of humans and SIV infection of Asian macaques, damage to the tight epithelial barrier of the gastrointestinal (GI) tract leads to microbial translocation, which contributes to chronic immune activation and disease progression (2). The increased mortality in ARV-treated, HIV-infected individuals is associated with inflammation and cardiovascular disease, which are in turn associated with GI mucosal damage and microbial translocation that do not resolve completely with ARV treatment $(1,3)$. Probiotic and prebiotic supplements have improved outcomes in several diseases characterized by GI tract inflammation (4-6), and probiotics have safely been administered to HIV-infected individuals resulting in modest improvements in $\mathrm{CD}^{+} \mathrm{T}$ cell counts and clinical GI symptoms even without ARV treatment (7-9). We therefore studied potential benefits of synbiotic supplementation of ARVs in SIV-infected Asian macaques.

\section{Results and Discussion}

Seven chronically SIV-infected pigtail macaques (PTM) received treatment with probiotics/prebiotics (PP) (VSL\#3, Culturelle; prebiotic inulin) for 60 days (Supplemental Figure 1; supplemental material available online with this article; doi:10.1172/ JCI66227DS1). These PP were chosen based on previous studies showing improved GI tract physiology and/or decreased inflammation $(5,6,10)$. PP treatment alone did not affect plasma viral loads or $\mathrm{CD}^{+} \mathrm{T}$ cell numbers in blood or the GI tract (Supplemental Figure 2). To evaluate potential PP-mediated benefits in

Conflict of interest: The authors have declared that no conflict of interest exists. Citation for this article: J Clin Invest. 2013;123(2):903-907. doi:10.1172/JCI66227. the absence of high viral replication, animals received ARVs in conjunction with PP (ARV+PP) for 5 months. Four animals were treated with ARVs alone for 5 months (Supplemental Figure 1). ARVs suppressed plasma viremia to less than 50 copy equivalents $/ \mathrm{ml}$ in 10 of 11 PTM studied (Supplemental Figure 2A); 1 PP-treated animal responded to ARVs slowly (indicated by square plot symbol in all figures). We evaluated longitudinal effects of PP treatment on the fecal microbiome (Supplemental Figure 3 and Supplemental Methods). Consistent with previous studies, we found no substantial SIV-associated alterations in the microbiome (11). Moreover, we only saw subtle changes in the composition of the fecal microbiota in animals treated with PP relative to animals treated with ARVs alone. However, several factors may decrease our ability to identify PP-mediated alterations in the microbiome. For example, fecal microbiome may not capture bacterial species adhering directly to GI tract epithelium; PP treatment may affect the microbiome in GI tissues not present in feces; PP may affect microbes capable of translocating; and our analysis may not have sequenced deep enough into the microbiome to observe subtle differences. However, it is clear that even very minor changes in the microbiota can have extensive effects in GI immunity (12).

We next measured blood $\mathrm{CD}^{+} \mathrm{T}$ cell counts. While we observed expected increases in $\mathrm{CD}^{+} \mathrm{T}$ cells after initiation of ARVs in both groups, there was no significant difference between ARV and ARV+PP groups (Supplemental Figure 2B). Thus, we focused our subsequent studies on the GI tract, the expected site of PP activity.

We assessed changes in expression of immune genes by microarray analysis on RNA isolated from colonic CD45+ leukocytes from our animals taken at necropsy. Distinct gene expression profiles were evident after heat map cluster analysis, with more than 200 genes differentially expressed (Figure 1A). Analysis of key differentially expressed genes demonstrated genes generally expressed by APCs being upregulated in ARV+PP animals (Fig- 

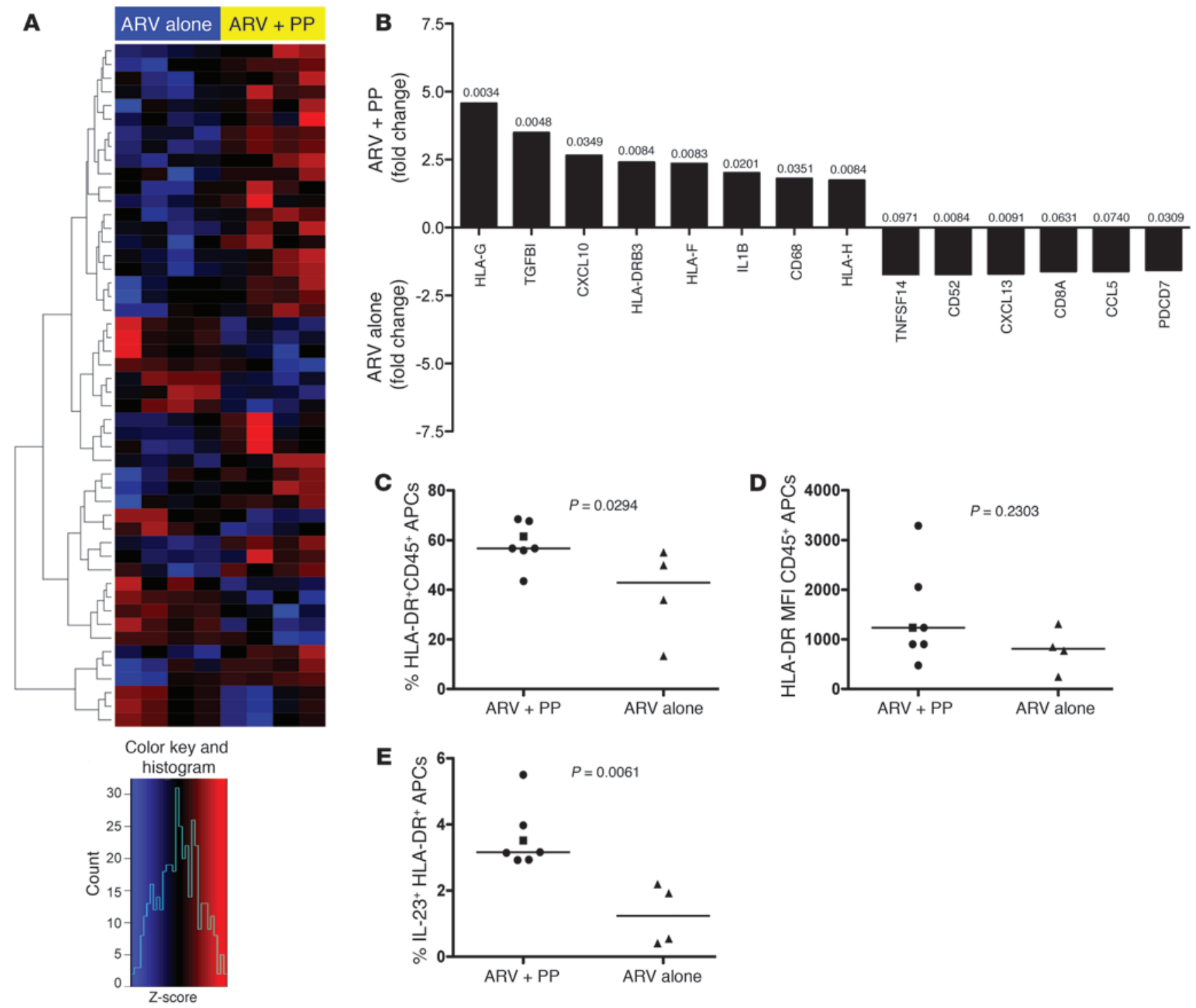

\section{Figure 1}

PP-treated animals have increased expression of APC-related genes. (A) Top 200 genes based on $P$ value for contrast between RNA extractions from colonic CD45+ leukocytes from animals treated with ARV alone (left) and animals treated with ARV+PP (right). The complete linkage method was used for hierarchical cluster analysis. Rows, normalized expression value for single genes; columns, each sample; red, upregulated; blue, downregulated gene expression based on Z-score (see legend below). (B) Fold change of selected upregulated genes in colonic leukocytes in animals treated with ARV+PP (top) or ARV alone (bottom). $P$ values are indicated over each bar. (C) Frequency of HLA-DR+CD45+, live APCs in the colon. (D) MFI of HLA-DR on live CD45+ APCs. (E) Percentages of live, HLA-DR+CD45+ APCs in the colon producing IL-23 after mitogenic stimulation. Circles, ARV+PP-treated PTM with suppressed viremia; squares, ARV+PP-treated slow responder PTM; triangles, ARV-alone-treated PTM. Horizontal bars represent median. All data analyzed from necropsy samples ( day 315 after SIV).

ure $1 \mathrm{~B})$. These APC-associated and upregulated genes included HLA-G, HLA-DRB3, HLA-H, CD14, and CD68 (Figure 1B). To assess whether the increased APC-related genes reflected increased numbers of APCs in the colon, we measured the frequency of total HLA-DR ${ }^{+}$APCs and found a significantly higher frequency in PP-treated animals $(P=0.0294$; Figure $1 C)$. To determine whether this was related to increased HLA-DR expression per cell, we calculated MFI of HLA-DR on APCs and found no difference $(P=0.2303$; Figure 1D). Thus, the increased expression of APCrelated genes in $\mathrm{PP}$-treated animals likely reflects increased numbers of APCs. Genes for the APC-related chemokines and cytokines CXCL10 and $I L-1 b$ were also upregulated in PP-treated animals, as was the regulatory cytokine TGF-B (Figure 1B). Consistent with increased functionality of colonic APCs in ARV+PPtreated PTM suggested by microarray analysis, we found an increased frequency of mitogenically stimulated APCs producing IL-23 (Figure 1E), a cytokine essential for promotion of IL-17/ IL-22-producing lymphocytes, which have been shown to be decreased in HIV/SIV-infected individuals (13-15).

Given the essential role of APCs in T cell immunity, we next assessed percentages and functionality of colonic CD4 ${ }^{+} \mathrm{T}$ cells. Indeed, we found an almost 2 -fold increase in the frequency of 

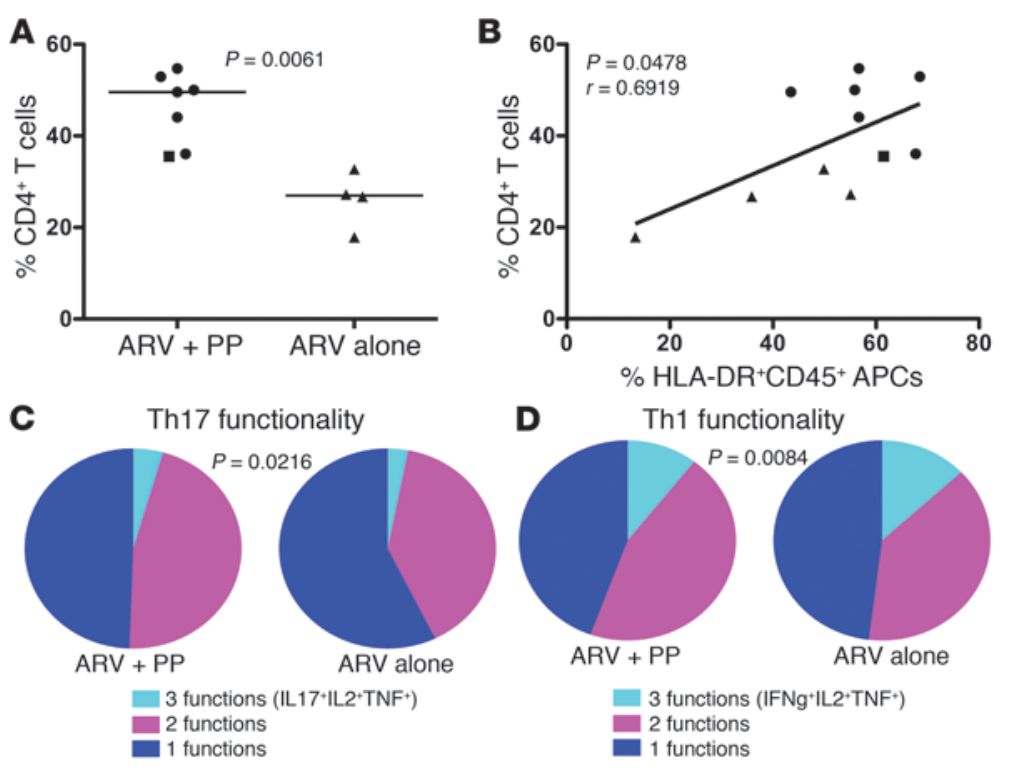

\section{Figure 2}

Enhanced reconstitution and functionality of CD4+ $T$ cells in the colon in PP-treated PTM. (A) Percentages of colon CD4+ $\mathrm{T}$ cells measured by flow cytometry. (B) Correlation between percentages of $\mathrm{HLA}-\mathrm{DR}{ }^{+} \mathrm{CD} 45^{+}$, live APCs in the colon compared with percentages of CD4+ $\mathrm{T}$ cells in the colon. (C and D) Pie charts represent fraction of cells producing 3 (cyan), 2 (magenta), or 1 (blue) cytokine(s) for Th17 cells (C) or Th1 cells (D) in the colon. (E) Percentages of $\mathrm{Ki}_{67}{ }^{+} \mathrm{CD} 4^{+} \mathrm{T}$ cells in the colon measured by flow cytometry. Circles, ARV+PP-treated PTM with suppressed viremia; squares, ARV+PP-treated slow responder PTM; triangles, ARV-alone-treated PTM. All data analyzed from necropsy samples ( day 315 after SIV). Horizontal bars represent median; diagonal lines represent linear regression.

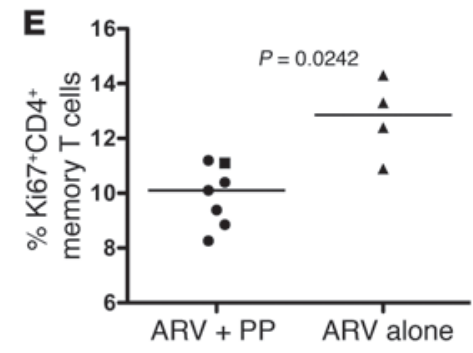

$\mathrm{CD}^{+} \mathrm{T}$ cells in the colon of PP-treated PTM (average 46.13\%) compared with PTM treated with ARVs alone (average 26.12\%) $(P=0.0061 ;$ Figure 2A). This increased reconstitution is important, considering that long-term ARV-treated, HIV-infected individuals rarely reconstitute GI tract $\mathrm{CD}^{+} \mathrm{T}$ cells to healthy levels (16), yet the ARV+PP animals substantially reconstituted colonic $\mathrm{CD}^{+}$ $\mathrm{T}$ cells to near normal levels with only 5 months of treatment. Moreover, the increased levels of colonic $\mathrm{CD}^{+} \mathrm{T}$ cells we observed in the ARV+PP animals were not simply due to higher levels of $\mathrm{CD}^{+} \mathrm{T}$ cells prior to initiation of therapy. Although longitudinal colon specimens were not possible, massive depletion of $\mathrm{CD}^{+}$ $\mathrm{T}$ cells occurs throughout the GI tract (17) and we were able to evaluate longitudinal $\mathrm{CD}^{+} \mathrm{T}$ cell percentages in endoscopically obtained jejunal biopsies (Supplemental Figure 4) demonstrating comparable massive depletion of GI tract $\mathrm{CD}^{+} \mathrm{T}$ cells in both groups of SIV-infected PTM prior to ARVs. The enhanced reconstitution of GI tract $\mathrm{CD}^{+}{ }^{+} \mathrm{T}$ cells, but not peripheral $\mathrm{CD}^{+}$ $\mathrm{T}$ cells, after PP treatment is consistent with previous research demonstrating that $\mathrm{ARV}$ treatment alone reconstitutes blood, but not mucosal, $\mathrm{CD}^{+} \mathrm{T}$ cells, an effect possibly attributable to lack of mucosal homing (18). Thus, the lack of increased CD4 ${ }^{+} \mathrm{T}$ cells in the blood of ARV+PP animals compared with ARV-alone animals could be due to cellular homing to the GI tract and thus decreasing the pool that may otherwise circulate in blood. A potential mechanism underlying this homing could be the alteration in the local GI tract APCs of PP-treated animals. Indeed, we observed a significant correlation between the frequency of APCs and $\mathrm{CD}^{+}$ $\mathrm{T}$ cells in the colon $(P=0.0478$; Figure $2 \mathrm{~B})$.
Increases in multifunctional $\mathrm{T}$ cells are associated with slower HIV disease progression and increased reconstitution of GI CD4 ${ }^{+}$ T cells after ARV administration (19). Thus, with enhanced reconstitution of colonic $\mathrm{CD} 4^{+} \mathrm{T}$ cells in the $\mathrm{ARV}+\mathrm{PP}$-treated animals, we assessed multifunctionality in colonic $\mathrm{CD}^{+} \mathrm{T}$ cells after mitogenic stimulation. Multifunctional Th17 cells were defined as producing 3 cytokines (IL-17, TNF- $\alpha$, and IL-2) or 2 cytokines (IL-17 and either TNF- $\alpha$ or IL-2), while monofunctional cells produced only IL-17. Consistent with increased production of IL-23 by APCs, which promotes Th17 responses (Figure 1E), we found that PP-treated animals had significantly higher percentages of multifunctional Th17 cells in the colon compared with animals treated with ARVs alone $(P=0.0216$; Figure $2 \mathrm{C})$. We also assessed multifunctionality in colon memory CD $4^{+}$Th 1 cells (IFN- $\gamma$, TNF- $\alpha$, and IL-2) and found significantly increased percentages of multifunctional Th1 cells $(P=0.0084$; Figure 2D) in PP-treated PTM. Thus, PP treatment enhanced functionality of GI tract CD4 ${ }^{+}$ T cells, potentially due to increased APCs.

Given that the strongest predictor of disease progression in untreated HIV infection is the degree of immune activation (20) and that persistent immune activation despite long term ARV treatment is associated with increased morbidity and mortality (1), we measured immune activation by expression of Ki67 among colonic memory $\mathrm{CD}^{+} \mathrm{T}$ cells. We found decreased immune activation of colonic CD4 ${ }^{+} \mathrm{T}$ cells in PP-treated PTM compared with ARV-alone-treated PTM $(P=0.0242$; Figure $2 \mathrm{E})$. Consistent with decreased immune activation in the GI tract, we also observed a trend toward decreased plasma levels of D-dimers in PP-treated 


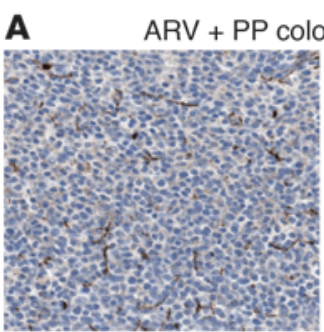

PTGC45

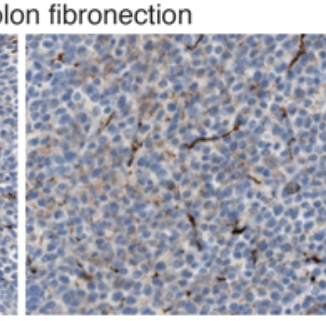

PTGP27
B

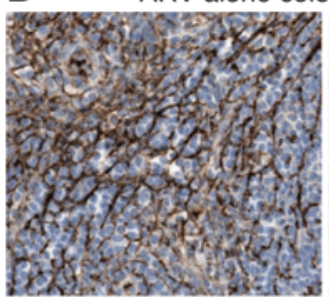

PTA2P032

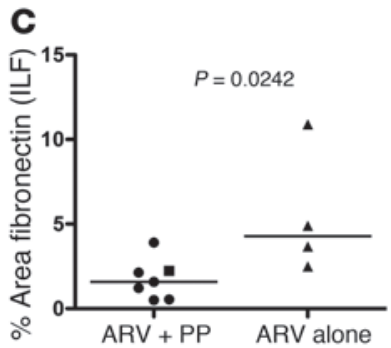

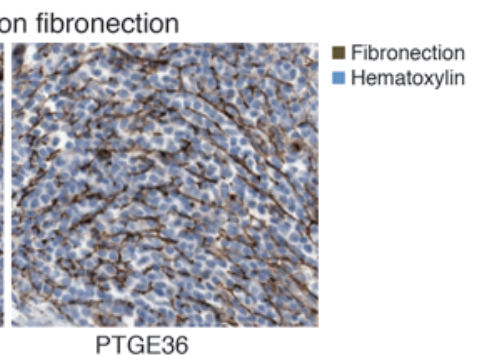

PTGE36

D

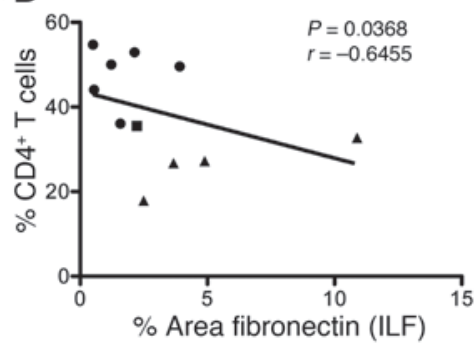

- Fibronection = Hematoxylin

\section{Figure 3}

PP treatment reduces fibrosis and is associated with increased CD4 ${ }^{+} \mathrm{T}$ cells. ( $\mathrm{A}$ and $\mathbf{B}$ ) Representative immunohistochemical staining of fibronectin in colonic isolated lymphoid follicles (ILF). Original magnification, $\times 250$. (A) Animals treated with ARV+PP. (B) Animals treated with ARV alone. (C) Percentage area of fibronectin in colon ILF by quantitative image analysis. (D) The fraction of colon fibronectin versus colonic CD4+ $\mathrm{T}$ cell percentages. Circles, ARV+PPtreated PTM with suppressed viremia; square, ARV+PP-treated slow responder PTM; triangles, ARV-alone-treated PTM. All data analyzed from necropsy samples ( day 315 after SIV). Horizontal bars represent median; diagonal lines represent linear regression. animals $(P=0.1167$, data not shown). This is particularly important given that $\mathrm{D}$-dimer levels represent an inflammatory biomarker associated with the clotting cascade and elevated D-dimer levels are associated with cardiovascular disease in ARV-treated, HIV-infected individuals $(1,3)$. We found no significant changes in peripheral immune activation or microbial translocation in the 5 months of treatment (Supplemental Figure 5), and future longterm studies on synbiotic treatments during HIV and SIV infection may be necessary.

Limited reconstitution of GI tract $\mathrm{CD}^{+} \mathrm{T}$ cells in long-term ARV-treated HIV-infected individuals has been attributed, at least in part, to fibrosis within GI tract lymphoid follicles (21). Thus, we measured fibrosis in lymphoid follicles by immunohistochemical staining of fibronectin (Figure 3, A and B). We found significantly reduced fibrosis in $\mathrm{ARV}+\mathrm{PP}$ animals compared with ARV-only animals $(P=0.0242$; Figure $3 C)$. Furthermore, we found a negative correlation between fibrosis in the colon and the frequency of colonic CD4 ${ }^{+} \mathrm{T}$ cells $(P=0.0368, \mathrm{r}=-0.6455$ Figure $3 \mathrm{D})$ Thus, a potential mechanism underlying the increased frequency of $\mathrm{CD}^{+}$ $\mathrm{T}$ cells in the colon in PP-treated animals may be resolution of fibrosis in the GI tract.

In summary, here we demonstrate that symbiotic PP supplementation of ARV treatment enhanced GI immune function and increased reconstitution of colonic $\mathrm{CD}^{+} \mathrm{T}$ cells, possibly by increasing APC frequency and/or function and decreasing inflammation associated fibrosis. PP provide an exciting adjunctive therapeutic approach for HIV infection, as they are well tolerated and inexpensive $(7,22)$. While additional studies will be required to extend the present findings to HIV infection, consistent with previous stud- ies $(7,8,22)$, our data suggest that PP treatment may be a useful approach to supplementing ARV therapy in HIV-infected individuals to mitigate residual GI inflammation and damage, thereby potentially having a beneficial impact on morbidity and mortality.

\section{Methods}

Animals. Eleven PTM (Macaca nemestrina) were infected with 3000 TCID50 of SIVmac239 i.v. At day 100 after infection, 7 PTM (ARV+PP group) were given probiotics VSL\#3 $\left(2.25 \times 10^{11}\right.$ bacteria daily) and Culturelle $\left(1 \times 10^{10}\right.$ bacteria and $200 \mathrm{mg}$ inulin daily) for 60 days. At day 160, combination ARV therapy was initiated in all 11 PTM $(20-30 \mathrm{mg} / \mathrm{kg}$ PMPA, $30 \mathrm{mg} / \mathrm{kg}$ FTC once daily, s.c., and $120 \mathrm{mg} \mathrm{L812,} 50 \mathrm{mg}$ L564 twice daily, oral) for 5 months (Supplemental Figure 1). Animals were euthanized, and blood and GI tissues were processed into single-cell suspensions (23) and paraffin embedded for immunohistochemistry. Four PTM expressed the MHC allele Mane-A $1 * 084$; however, none of these animals had undetectable plasma viral loads or increased $\mathrm{CD} 4^{+} \mathrm{T}$ cell reconstitution after treatment (Supplemental Figure 6).

Microarray analysis. CD45+ (clone MB4-6D6) leukocytes were sorted from colon samples from $4 \mathrm{ARV}+\mathrm{PP}$ animals and $4 \mathrm{ARV}$-alone animals. RNA samples were hybridized to the Illumina HumanHT-12 version 4 Expression BeadChip and quantified using an Illumina iScan System. Genes with significant differential expression levels were identified using the Bioconductor Limma Package with 1.5-fold or more change and a raw $P$ value of less than 0.05 and corrected for multiple comparison as previously described (15) (GEO GSE42232).

Flow cytometry. Multicolor flow cytometric analysis was performed on samples as previously described (23) using crossreactive anti-human or anti-nonhuman primate antibodies. 
Immunohistochemistry. Immunohistochemical staining was performed as previously described $(21,23)$ on paraformaldehyde-fixed tissues with mouse anti-fibronectin (clone FBN11; Lab Vision) Stained slides were scanned at $\times 200$ magnification using the ScanScope CS System (Aperio Technologies, Inc.) yielding high-resolution data for the entire tissue section.

Viral loads. Plasma viral RNA levels were determined by RT-PCR from SIVmac239-inoculated PTM as previously described (24).

Statistics. $P$ values for microarray analysis were determined using a linear model empirical Bayes method corrected for multiple comparisons, by partial permutation test between all samples in each treatment group for multifunctional analysis (described in ref. 25), and by Mann-Whitney $t$ tests for all other cases. $R$ values were calculated from Spearman correlations.

Study approval. Animals were housed and cared for in accordance with standards of the American Association for Accreditation of Laboratory Animal Care (AAALAC) in AAALAC-accredited facilities, and all animal procedures were performed according to protocols approved by the Institutional Animal Care and Use Committees of NIAID/NIH.

\section{Acknowledgments}

We acknowledge Heather Cronise, JoAnne Swerczek, Richard Herbert, and all veterinary staff at the NIH Animal Center. RNA isolation, microarrays, and bioinformatic analysis was provided by the Collaborative Genomics Center (CGC) at VGTI-Florida. We thank Cleveland Immunopathogenesis Consortium/Bad Boys of Cleveland (CLIC/BBC) for helpful discussions. Studies were supported by the Intramural NIAID, US NIH program, and with federal funds from the National Cancer Institute (NCI)/ NIH, contract HHSN261200800001E. Histology support was provided by the Pathology/Histotechnology Laboratory service of the NCI. The content of this publication does not necessarily reflect the views or policies of the Department of Health and Human Services (DHHS), nor does the mention of trade names, commercial products, or organizations imply endorsement by the US Government.

Received for publication August 7, 2012, and accepted in revised form November 13, 2012.

Address correspondence to: Jason M. Brenchley, 9000 Rockville Pike, Building 4/Room 201, Bethesda, Maryland 20892, USA. Phone: 301.496.1498; Fax: 301.402.0226; E-mail: jbrenchl@ mail.nih.gov.
1. Rodger AJ, et al. Activation and coagulation biomarkers are independent predictors of the development of opportunistic disease in patients with HIV infection. J Infect Dis. 2009;200(6):973-983.

2. Estes JD, et al. Damaged intestinal epithelial integrity linked to microbial translocation in pathogenic simian immunodeficiency virus infections. PLoS Pathog. 2010;6(8):e1001052.

3. Sandler NG, et al. Plasma levels of soluble CD14 independently predict mortality in HIV infection. J Infect Dis. 2011;203(6):780-790.

4. Marteau PR, de Vrese M, Cellier CJ, Schrezenmeir J. Protection from gastrointestinal diseases with the use of probiotics. Am J Clin Nutr. 2001; 73(2 suppl):430S-436S.

5 . Madsen KL. The use of probiotics in gastrointestinal disease. Can J Gastroenterol. 2001;15(12):817-822.

6. Heilpern D, Szilagyi A. Manipulation of intestinal microbial flora for therapeutic benefit in inflammatory bowel diseases: review of clinical trials of probiotics, pre-biotics and synbiotics. Rev Recent Clin Trials. 2008;3(3):167-184.

7. Hummelen R, et al. Effect of 25 weeks probiotic supplementation on immune function of HIV patients. Gut Microbes. 2011;2(2):80-85.

8. Irvine SL, Hummelen R, Hekmat S. Probiotic yogurt consumption may improve gastrointestinal symptoms, productivity, and nutritional intake of people living with human immunodeficiency virus in Mwanza, Tanzania. Nutr Res. 2011;31(12):875-881.
9. Schunter M, et al. Randomized pilot trial of a synbiotic dietary supplement in chronic HIV-1 infection. BMC Complement Altern Med. 2012;12:84.

10. Dai C, Zhao DH, Jiang M. VSL\#3 probiotics regulate the intestinal epithelial barrier in vivo and in vitro via the $\mathrm{p} 38$ and ERK signaling pathways. Int J Mol Med. 2012;29(2):202-208.

11. McKenna $P$, et al. The macaque gut microbiome in health, lentiviral infection, and chronic enterocolitis. PLoS Pathog. 2008;4(2):e20.

12. Costello EK, Lauber CL, Hamady M, Fierer N, Gordon JI, Knight R. Bacterial community variation in human body habitats across space and time. Science. 2009;326(5960):1694-1697.

13. Aggarwal S, Ghilardi N, Xie MH, de Sauvage FJ, Gurney AL. Interleukin-23 promotes a distinct CD4 T cell activation state characterized by the production of interleukin-17. JBiol Chem. 2003;278(3):1910-1914.

14. Klatt NR, Brenchley JM. Th17 cell dynamics in HIV infection. Curr Opin HIV AIDS. 2010;5(2):135-140.

15. Klatt NR, et al. Loss of mucosal CD103+ DCs and IL-17+ and IL-22+ lymphocytes is associated with mucosal damage in SIV infection. Mucosal Immunol. 2012;5(6):646-657.

16. Mehandru S, et al. Lack of mucosal immune reconstitution during prolonged treatment of acute and early HIV-1 infection. PLoS Med. 2006;3(12):e484.

17. Veazey RS, et al. Gastrointestinal tract as a major site of CD4+ T cell depletion and viral replication in SIV infection. Science. 1998;280(5362):427-431.
18. Mavigner M, et al. Altered CD4+ T cell homing to the gut impairs mucosal immune reconstitution in treated HIV-infected individuals. J Clin Invest. 2011;122(1):62-69.

19. Shacklett BL, Ferre AL. Mucosal immunity in HIV controllers: the right place at the right time. Curr Opin HIV AIDS. 2011;6(3):202-207.

20. Giorgi JV, et al. Shorter survival in advanced human immunodeficiency virus type 1 infection is more closely associated with $\mathrm{T}$ lymphocyte activation than with plasma virus burden or virus chemokine coreceptor usage. J Infect Dis. 1999;179(4):859-870.

21. Estes J, et al. Collagen deposition limits immune reconstitution in the gut. J Infect Dis. 2008; 198(4):456-464

22. Hummelen R, et al. Effect of micronutrient and probiotic fortified yogurt on immune-function of anti-retroviral therapy naive HIV patients. Nutrients. 2011;3(10):897-909.

23. Klatt NR, et al. Compromised gastrointestinal integrity in pigtail macaques is associated with increased microbial translocation, immune activation, and IL-17 production in the absence of SIV infection. Mucosal Immunol. 2010;3(4):387-398.

24. Klatt NR, et al. Dynamics of simian immunodeficiency virus SIVmac239 infection in pigtail macaques. J Virol. 2011;86(2):1203-1213.

25. Roederer M, Nozzi JL, Nason MC. SPICE: exploration and analysis of post-cytometric complex multivariate datasets. Cytometry A. 2011;79(2):167-174. 